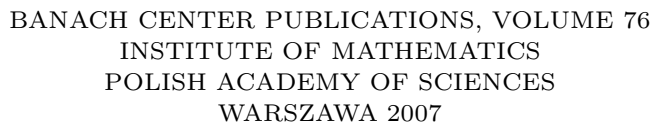

\title{
THE WORKS OF CHARLES EHRESMANN ON CONNECTIONS: FROM CARTAN CONNECTIONS TO CONNECTIONS ON FIBRE BUNDLES
}

\author{
CHARLES-MICHEL MARLE \\ Institut de Mathématiques, Université Pierre et Marie Curie, \\ 4, place Jussieu, 75252 Paris cédex 05, France \\ E-mail: charles-michel.marle@polytechnique.org
}

\begin{abstract}
Around 1923, Élie Cartan introduced affine connections on manifolds and defined the main related concepts: torsion, curvature, holonomy groups. He discussed applications of these concepts in Classical and Relativistic Mechanics; in particular he explained how parallel transport with respect to a connection can be related to the principle of inertia in Galilean Mechanics and, more generally, can be used to model the motion of a particle in a gravitational field. In subsequent papers, Élie Cartan extended these concepts for other types of connections on a manifold: Euclidean, Galilean and Minkowskian connections which can be considered as special types of affine connections, the group of affine transformations of the affine tangent space being replaced by a suitable subgroup; and more generally, conformal and projective connections, associated to a group which is no more a subgroup of the affine group.

Around 1950, Charles Ehresmann introduced connections on a fibre bundle and, when the bundle has a Lie group as structure group, connection forms on the associated principal bundle, with values in the Lie algebra of the structure group. He called Cartan connections the various types of connections on a manifold previously introduced by É. Cartan, and explained how they can be considered as special cases of connections on a fibre bundle with a Lie group $G$ as structure group: the standard fibre of the bundle is then an homogeneous space $G / G^{\prime}$; its dimension is equal to that of the base manifold; a Cartan connection determines an isomorphism of the vector bundle tangent to the the base manifold onto the vector bundle of vertical vectors tangent to the fibres of the bundle along a global section.
\end{abstract}

These works are reviewed and some applications of the theory of connections are sketched.

2000 Mathematics Subject Classification: Primary53C05; Secondary 53B05, 53B10, 53B15, $70 \mathrm{G} 45$.

Key words and phrases: Cartan connections, principle of inertia.

The paper is in final form and no version of it will be published elsewhere. 
1. Introduction. It is a great honor for me to be invited at the Seventh International Conference on Geometry and Topology of Manifolds, dedicated to the mathematical legacy of Charles Ehresmann. I enjoyed with great pleasure the hospitality of the Mathematical Research and Conference Center of the Polish Academy of Sciences, and I address my warmest thanks to the organizers and to the supporting institutions.

Around 1923, Élie Cartan [1, 2,3] introduced the notion of an affine connection on a manifold. That notion was previously used, in a less general setting, by H. Weyl [20] and rests on the idea of parallel transport due to T. Levi-Civita [14].

At the very beginning of [1], even before defining explicitly affine connections, Élie Cartan explains how that concept can be used in Newtonian and Einsteinian Mechanics. He shows that the principle of inertia (which is at the foundations of Mechanics), according to which a material point particle, when no forces act on it, moves along a straight line with a constant velocity, can be expressed locally by the use of an affine connection. Under that form, that principle remains valid in (curved) Einsteinian space-times.

Cartan even shows that by a suitable adjustment of the connection, a gravity force (that means, an acceleration field) can be accounted for, and becomes a part of the Geometry of space-time. That result expresses the famous equivalence principle used by Einstein for the foundations of General Relativity. As shown by Cartan, it is valid for Newtonian Mechanics as well.

Then Élie Cartan presents a thorough geometric study of affine connections; he defines their curvature and torsion, and discusses the parallel displacement of a frame along a closed loop. He introduces Euclidean, Galilean and Minkowskian connections, for which the group of affine transformations is replaced by a suitable subgroup. In $[4,5,6]$ he introduces more general types of connections associated to transformation groups which are no more subgroups of the group of affine transformations.

Cartan's ideas were fully formalized by Charles Ehresmann in the framework of connections on fibre bundles, which he introduced in [8].

In Section 2 we briefly present Cartan's intuitive ideas about connections. Then in Section 3 we describe Ehresmann connections on fibre bundles, and in Section 4 Cartan connections as seen by Ehresmann. In Section 5 we present with more details examples of Cartan connections, including affine, projective and conformal connections. In Section 6, following Cartan, we show how a gravitational force can be included in the geometry of space-time by the use of a suitable connection, and we briefly present other applications of connections: Geometric quantization, phases in mechanics, nonholonomic or active constraints, Maxwell's equations, Yang-Mills fields.

2. Cartan affine connections and their generalizations. É. Cartan writes in [1]: "Une variété à connexion affine est une variété qui, au voisinage immédiat de chaque point, a tous les caractères d'un espace affine, et pour laquelle on a une loi de repérage des domaines entourant deux points infiniment voisins : cela veut dire que si, en chaque point, on se donne un système de coordonnées cartésiennes ayant ce point pour origine, on connaît les formules de transformation (de même nature que dans l'espace affine) 
qui permettent de passer d'un système de référence à tout autre système de référence d'origine infiniment voisine".

Approximate translation: "A manifold with an affine connection is a manifold whose properties, in the neighborhood of each point, are those of an affine space, and on which there is a law for fitting together the neighborhoods of two infinitesimally nearby points: it means that if, on a neighborhood of each point, we have chosen Cartesian coordinates with that point as origin, we know the transformation formulae (of the same nature as those valid in an affine space) which allow to go from a reference frame to another reference frame with an infinitesimally nearby origin".

In fact, given a smooth manifold $M$, it is not on a neighborhood of each point $m \in M$ that Cartan considers a local affine structure. Rather, at each point $m \in M$, he considers the tangent space $T_{m} M$ endowed with its natural affine space structure. And he writes: "La variété sera dite à connexion affine lorsqu'on aura défini, d'une manière d'ailleurs arbitraire, une loi permettant de repérer l'un par rapport à l'autre les espaces affines attachés à deux points infiniment voisins quelconques $m$ et $m^{\prime}$ de la variété; cette loi permettra de dire que tel point de l'espace affine attaché au point $m^{\prime}$ correspond à tel point de l'espace affine attaché au point $m$, que tel vecteur du premier espace est parallèle ou équipollent à tel vecteur du second espace. En particulier le point $m^{\prime}$ lui-même sera repéré par rapport à l'espace affine du point $m$...".

Approximate translation: "The manifold will be said to be endowed with an affine connection once we have defined, in an arbitrary way, a law allowing to localize one with respect to the other the affine spaces attached to two infinitesimally nearby points $m$ and $m^{\prime}$ of that manifold; that law will tell us which point of the affine space attached to $m^{\prime}$ corresponds to a given point of the space attached to $m$, and will tell us whether a vector living in the first space is parallel, or equipollent, to a vector living in the second space; in particular, the point $m^{\prime}$ itself will be localized in the affine space attached to $m$...".

Cartan then explains with more details how to define an affine connection on a 3dimensional manifold $M$; of course his definition extends easily to manifolds of any dimension. He considers, at each point $m \in M$, an affine frame of the affine tangent space $T_{m} M$, with as origin the point $m$ itself (identified with the null vector at $m$ ), and with the linear basis $\left(e_{1}, e_{2}, e_{3}\right)$ as basis. In order to define the law which links the affine spaces tangent to the manifold $M$ at two infinitesimally nearby points $m$ and $m^{\prime}$, he write the relations

$$
\begin{aligned}
d m & =\omega^{1} e_{1}+\omega^{2} e_{2}+\omega^{3} e_{3}, \\
d e_{i} & =\omega_{i}^{1} e_{1}+\omega_{i}^{2} e_{2}+\omega_{i}^{3} e_{3}, \quad i=1,2,3 .
\end{aligned}
$$

These equations mean that the point $m^{\prime}$, origin of $T_{m^{\prime}} M$, infinitesimally near $m$, must be identified with the point

$$
m+\omega^{1} e_{1}+\omega^{2} e_{2}+\omega^{3} e_{3}
$$

of the affine space $T_{m} M$.

Similarly, the vectors $e_{1}^{\prime}, e_{2}^{\prime}, e_{3}^{\prime}$ of $T_{m^{\prime}} M$ must be identified with the vectors

$$
e_{i}^{\prime}=e_{i}+\omega_{i}^{1} e_{1}+\omega_{i}^{2} e_{2}+\omega_{i}^{3} e_{3}, i=1,2,3
$$


of $T_{m} M$.

Equations (1) must be understood as equalities between differential 1-forms on a 15-dimensional space with, as coordinates, the 3 coordinates which specify a point on $M$, and 12 more coordinates on which depend the affine frames of a 3-dimensional affine space. In fact, these differential 1-forms live on the principal bundle of affine frames of the affine tangent spaces to the maniflold $M$. The meaning of Equations (1) is the following: let $h=\left(m, e_{1}, e_{2}, e_{3}\right)$ be an affine frame of the affine tangent space $T_{m} M$ and let $V$ be a vector tangent at $h$ to the space of affine frames. The infinitesimal displacement of that affine frame defined by the vector $V$ is parallel, with respect to the connection, if and only if $\omega(V)=0$, where $\omega$ is the 1-form of the connection (in the sense of Ehresmann, as introduced later). Equation $\omega(V)=0$, when explicitly written, leads to the 12 scalar equations (1) because the 1-form $\omega$ takes its values in the 12-dimensional Lie algebra of the group of affine transformations of $\mathbb{R}^{3}$. This will become clear with the works of Charles Ehresmann [8].

Euclidean connections, Galilean connections, Minkowskian connections, ..., considered by Cartan, appear as special cases of affine connections, obtained by replacing the group of affine transformations of an affine space by an appropriate subgroup. For example, on a 3 -dimensional manifold $M$ endowed with a Riemannian metric $g$, instead of general affine frames $h=\left(m, e_{1}, e_{2}, e_{3}\right)$ with the contact point $m$ as origin of the affine tangent space $T_{m} M$, Cartan considers only orthonormal frames, which satisfy

$$
g\left(e_{i}, e_{j}\right)=\delta_{i j} .
$$

Now the dimension of the space of orthonormal affine frames is 9 (instead of 15 for the space of general affine frames). By differentiation of the above relations we see that the 1 forms $\omega^{i}$ and $\omega_{i}^{j}$ of Equations (1) must satisfy

$$
\omega_{1}^{1}=\omega_{2}^{2}=\omega_{3}^{3}=0, \quad \omega_{2}^{3}+\omega_{3}^{2}=0, \quad \omega_{3}^{1}+\omega_{1}^{3}=0, \quad \omega_{1}^{2}+\omega_{2}^{1}=0 .
$$

Only 6 of the 12 scalar equations (1) are now independent (in agreement with the dimension, 6 , of the Lie algebra of the group of affine orthogonal transformations of $\mathbb{R}^{3}$ ).

Other types of connections were defined and discussed by É. Cartan $[4,5,6]$, with transformation groups which are no more subgroups of the group of affine transformations: conformal connections, projective connections, ... The general idea underlying the notions of such connections on a manifold $M$ is to use, as a local model of that manifold, an homogeneous space of the same dimension as $M$. A copy of that homogeneous space is attached at each point of the manifold, and considered as "tangent" at that point to the manifold. The connection is essentially a law which indicates how these homogeneous spaces are glued together.

Of course, affine connections appear as the special case in which the homogeneous space which is attached to each point of the manifold is an affine space, more precisely the affine space tangent to the manifold at that point.

For conformal connections on an $n$-dimensional manifold, É. Cartan [4] writes: "Attachons à chaque point $P$ de cette variété un espace conforme à $n$ dimensions, . . La variété sera dite à connexion conforme si nous nous donnons une loi (d'ailleurs arbitraire) per- 
mettant de rapporter, d'une manière conforme, l'espace conforme attaché au point $P$ de la variété à l'espace conforme attaché au point infiniment voisin $P^{\prime}$ ".

Approximate translation: "Let us link an $n$-dimensional conformal space to each point $P$ of our manifold. That manifold will be said to be endowed with a conformal connection when we have specified, in an arbitrary way, how to tie (or maybe identify) the conformal space linked at point $P$ with the conformal space linked to the infinitesimally nearby point $P^{\prime \prime}$.

In [4], Cartan writes: "L'idée fondamentale se rattache à la notion de parallélisme que M. T. Levi-Civita a introduite de manière si féconde. Les nombreux auteurs qui ont généralisé la théorie des espaces métriques sont tous partis de l'idée fondamentale de M. Levi-Civita, mais, semble-t-il, sans pouvoir la détacher de l'idée de vecteur. Cela n'a aucun inconvénient quand il s'agit de variétés à connexion affine ... Mais cela semblait interdire tout espoir de fonder une théorie autonome de variétés à connexion conforme ou projective. En fait, ce qu'il y a d'essentiel dans l'idée de M. Levi-Civita, c'est qu'elle donne un moyen pour raccorder entre eux deux petits morceaux infiniment voisins d'une variété, et c'est cette idée de raccord qui est féconde".

Approximate translation: "The fundamental idea stems from the notion of parallelism introduced by M. T. Levi-Civita in such a fruitful way. The many authors who genralized the theory of metric spaces all started from the fundamental idea of M. Levi-Civita, but, seemingly, without freeing it from the idea of vector. That does not matter as long as one deals with manifolds with affine connections ... But that seemed to forbid any hope to build an autonomous theory of manifolds with conformal or projective connections. In fact, the main thing in M. Levi-Civita's idea is that it allows to glue together two small, infinitesimally nearby pieces of a manifold, and it is that idea of gluing which is most fruitful".

3. Ehresmann connections. Let $E(B, F)$ be a locally trivial smooth fibre bundle with base $B$, standard fibre $F$, and canonical projection $\pi_{E}: E \rightarrow B$. For each $x \in B$, the fibre at $x, E_{x}=\pi_{E}^{-1}(x)$, is diffeomorphic to $F$. Ehresmann [8] defines an infinitiesimal connection on that bundle as follows.

Definition 1. An infinitesimal connection on the locally trivial bundle $E(B, F)$ is a vector sub-bundle $C$ of $T E$, complementary to $\operatorname{ker}\left(T \pi_{E}\right)$, i.e. such that for each $z \in E$,

$$
T_{z} E=\operatorname{ker}\left(T_{z} \pi_{E}\right) \oplus C_{z},
$$

which satisfies the additional condition:

$(C)$ Given any smooth path $t \mapsto x(t)$ in $B$ going from a point $x_{0}=x\left(t_{0}\right)$ to another point $x_{1}=x\left(t_{1}\right)$, and any $z_{0} \in E_{x_{0}}$, there exists a smooth path $t \mapsto z(t)$ in $E$, called the horizontal lift of $t \mapsto x(t)$ through $z_{0}$, such that

$$
z\left(t_{0}\right)=z_{0}, \quad \pi_{E}(z(t))=x(t) \text { and } \frac{d z(t)}{d t} \in C_{z(t)} \quad \text { for all } t \in\left[t_{0}, t_{1}\right] .
$$

More generally any smooth path $t \mapsto z(t)$ in $E$ is said to be horizontal if for each $t$, $\frac{d z(t)}{d t} \in C_{z(t)}$. 
REMARK 1. Under the assumptions of Definition 1, let $t \mapsto x(t)$ be a smooth path in $B$, defined on an interval $I$. For each $t_{0} \in I$ and each $z_{0} \in E_{x\left(t_{0}\right)}$, its horizontal lift $t \mapsto z(t)$ through $z_{0}$ is unique and defined on $I$. For each pair $\left(t_{0}, t_{1}\right) \in I^{2}$, the map $\varphi_{t_{1} t_{0}}: E_{x\left(t_{0}\right)} \rightarrow E_{x\left(t_{1}\right)}$

$$
z_{0} \mapsto \varphi_{t_{1} t_{0}}\left(z_{0}\right)=z\left(t_{1}\right)
$$

is a diffeomorphism of the fibre $E_{x\left(t_{0}\right)}$ onto the fibre $E_{x\left(t_{1}\right)}$, called the parallel transport of the fibres of $E(B, F)$ along the smooth path $t \mapsto x(t)$. By looking only at paths defined on finite closed intervals, and taking their end points, we see that the connection $C$ determines a homomorphism of the groupoid of smooth paths in $B$ which joint two points in $B$ into the groupoid of diffeomorphisms of a fibre or $E(B, F)$ onto another fibre.

Definition 2. Let $t \mapsto z(t)$ be a smooth path, defined on an interval $I$, with values in the total space $E$ of a locally trivial, smooth bundle $E(B, F)$ equipped with a connection $C$. Let $t \mapsto x(t)=\pi_{E}(z(t))$ be the corresponding smooth path in the base manifold $B$. Let $t_{0} \in I$ and, for each $t$, let $\varphi_{t t_{0}}$ be the parallel transport of the fibre $E_{x\left(t_{0}\right)}$ onto the fibre $E_{x(t)}$ along the path $t \mapsto x(t)$. The smooth path in the fibre $E_{x\left(t_{0}\right)}$,

$$
t \mapsto \varphi_{t t_{0}}^{-1} \circ z(t)
$$

is called the development of the path $t \mapsto z(t)$ in the fibre $E_{x\left(t_{0}\right)}$.

REMARK 2. The development in $E_{x\left(t_{0}\right)}$ of a path $t \mapsto z(t)$ in $E$ is a constant path if and only if $t \mapsto z(t)$ is horizontal.

We assume now that $E(B, F)$ is a smooth fibre bundle $E(B, F, G, H)$ with a Lie group $G$ as structure group. Let us first recall that notion, due to Ehresmann, and explain the notations. For simplicity we consider only smooth bundles, although in [7] Ehresmannn, more generally, defines topological bundles. The total space $E$ and the base $B$ of the bundle are two smooth manifolds, and there is a smooth submersion $\pi_{E}: E \rightarrow B$ of the total space onto the base. For each $x \in B, E_{x}=\pi_{E}^{-1}(x)$ is a smooth submanifold of $E$ called the fibre over $x$. The standard fibre $F$ is a smooth manifold and the structure group $G$ is a Lie group of diffeomorphisms of $F$, i.e. a Lie group $G$ which acts on the manifold $F$ on the left in such a way that the map $G \times F \rightarrow F,(g, f) \mapsto g f$, is smooth. That action is assumed to be effective, i.e. such that for each $g \in G$ other than the unit element $e$, the corresponding diffeomorphism $f \mapsto g f$ of the standard fibre $F$ is not the identity; therefore an element $g \in G$ is fully determined by the corresponding diffeomorphism of $F$. An atlas $\left(U_{i}, \varphi_{i}, i \in I\right)$ of the bundle $E(B, F, G, H)$ is defined by the following data:

(i) a family $\left(U_{i}, i \in I\right)$ of open subsets of $B$ such that $\bigcup_{i \in I} U_{i}=B$,

(ii) for each $i \in I$, a diffeomorphism

$$
\varphi_{i}: \pi_{E}^{-1}\left(U_{i}\right) \rightarrow U_{i} \times F
$$

such that for each $z \in \pi_{E}^{-1}\left(U_{i}\right)$,

$$
p_{1}\left(\varphi_{i}(z)\right)=\pi_{E}(z)
$$

(where $p_{1}: U_{i} \times F \rightarrow U_{i}$ is the first projection) and that for each pair $(i, j) \in I^{2}$ with $U_{i} \cap U_{j} \neq \emptyset$,

$$
\varphi_{j} \circ \varphi_{i}^{-1}(x, \xi)=\left(x, s_{j i}(x)(\xi)\right),
$$


with $x \in U_{i} \cap U_{j}, \xi \in F$, and where $s_{j i}: U_{i} \cap U_{j} \rightarrow G$ is a smooth map which takes its values in the Lie group $G$.

Two atlases $\left(U_{i}, \varphi_{i}, i \in I\right)$ and $\left(V_{j}, \psi_{j}, j \in J\right)$ of the bundle $E(B, F, G, H)$ are said to be equivalent if their union is still an atlas of that bundle. The $G$-bundle structure of $E(B, F, G, H)$ is determined by the choice of an equivalence class of atlases. Diffeomorphisms $\varphi_{i}: \pi_{E}^{-1}\left(U_{i}\right) \rightarrow U_{i} \times F$ which belong to an atlas of that equivalence class are called admissible local trivializations of the bundle $E(B, F, G, H)$.

For each $x \in B$, the fibre $E_{x}=\pi_{E}^{-1}(x)$ is diffeomorphic to $F$. More precisely there are admissible diffeomorphisms of $F$ onto $E_{x}$, obtained by means of an admissible local trivialization $\left(U_{i}, \varphi_{i}\right)$, with $x \in U_{i}$ :

$$
\xi \mapsto \varphi_{i}^{-1}(x, \xi), \quad \text { with } \xi \in F .
$$

For each $x \in E$, if $h: F \rightarrow \pi_{E}^{-1}(x)$ is an admissible diffeomorphism, another diffeomorphism $h^{\prime}: F \rightarrow \pi_{E}^{-1}(x)$ is admissible if and only if there exists $g \in G$ such that $h^{\prime}=h \circ g$.

In Ehresmann's notation $E(B, F, G, H)$, we have already explained what are the total space $E$, the base $B$, the standard fibre $F$ and the structure group $G$. We still have to define $H$ : it is the set of all admissible diffeomorphisms of the standard fibre $F$ onto the fibre $E_{x}=\pi_{E}^{-1}(x)$, for all $x \in B$. In [7], Ehresmann proves that $H$ is a smooth manifold. More precisely it is the total space of a smooth fibre bundle $H\left(B, G, G_{\gamma}, \bar{H}\right)$ with base $B$, standard fibre $G$, structure group $G_{\gamma}$ (that means the Lie group $G$ considered as acting on itself by left translations). The fibre $H_{x}=\pi_{H}^{-1}(x)$ over a point $x \in B$ is the set of admissible diffeomorphisms of $F$ onto $E_{x}=\pi_{E}^{-1}(x)$. In Ehresmann's notations $\bar{H}$ is the set of admissible diffeomorphisms $\bar{h}$ of the standard fibre $G$ onto the fibre $H_{x}$ of $H$ over some point $x \in B$. In fact, $\bar{H}$ can be identified with $H$, since an element $h \in H_{x}$, previously considered as a diffeomorphism of $F$ onto $E_{x}$, can also be considered as a diffeomorphism $\bar{h}$ of $G$ onto $H_{x}$ by writing

$$
\bar{h}(g)=h \circ g: F \rightarrow E_{x} .
$$

The smooth fibre bundle $H\left(B, G, G_{\gamma}, \bar{H}\right)$ is said to be a principal bundle because its structure group $G$ is also its standard fibre. More precisely, it is called the principal bundle associated to the bundle $E(B, F, G, H)$, and it is fully determined by that bundle.

Conversely, let $H\left(B, G, G_{\gamma}, \bar{H}\right)$ be a principal bundle with structure Lie group $G_{\gamma}$, and $F$ be a smooth manifold on which the Lie group $G$ acts on the left. There exist a bundle $E(B, F, G, H)$, unique up to an isomorphism, with structure Lie group $G$, standard fibre $F$ and $H\left(B, G, G_{\gamma}, \bar{H}\right)$ as associated principal bundle. The bundles obtained in that way are said to be associated to the given principal bundle $H\left(B, G, G_{\gamma}, \bar{H}\right)$.

REMARK 3. There is a natural, free action on the right of the structure Lie group $G$ on the total space $H$ of the principal bundle $H\left(B, G, G_{\gamma}, \bar{H}\right)$,

$$
H \times G \rightarrow H, \quad(h, g) \mapsto R_{g}(h)=h \circ g,
$$

whose orbits are the fibres $H_{x}, x \in B$. 
Definition 3. Let $E(B, F, G, H)$ be a fibre bundle with a Lie group $G$ as structure group. An infinitesimal connection $C$ on that bundle, in the sense of Definition 1, is said to be compatible with the structure group $G$, and is called a $G$-connection, if the parallel transport along any smooth path in $B$ starting from any point $x_{0} \in B$ and ending at any other point $x_{1} \in B$, is of the form $h_{1} \circ h_{0}^{-1}$, with $h_{0} \in H_{x_{0}}$ and $h_{1} \in H_{x_{1}}$.

Proposition 1. A G-connection on the bundle $E(B, F, G, H)$ determines a unique $G_{\gamma}$-connection $\bar{C}$ on the associated principal bundle $H\left(B, G, G_{\gamma}, \bar{H}\right)$ such that, for any smooth path $t \mapsto x(t)$ in the base $B$, any $t_{0}$ in the interval of definition of that path and any $h_{0} \in H_{x\left(t_{0}\right)}$, the horizontal lift through $h_{0}$ of the path $t \mapsto x(t)$ with respect to the connection $\bar{C}$ is the path

$$
t \mapsto h(t)=\varphi_{t t_{0}} \circ h_{0},
$$

where $\varphi_{t t_{0}}: E_{x\left(t_{0}\right)} \rightarrow E_{x(t)}$ is the parallel transport along the smooth path $t \mapsto x(t)$, for the $G$-connection $C$ on $E(B, F, G, H)$.

Conversely, a $G_{\gamma}$-connection $\bar{C}$ on the principal bundle $H\left(B, G, G_{\gamma}, \bar{H}\right)$ determines a unique $G$-connection $C$ on $E(B, F, G, H)$ such that, for any smooth path $t \mapsto x(t)$ in the base $B$, any $t_{0}$ in the interval of definition of that path and any $z_{0} \in E_{x\left(t_{0}\right)}$, the horizontal lift through $z_{0}$ of the path $t \mapsto x(t)$ with respect to the connection $C$ is the path

$$
t \mapsto z(t)=h(t)(\xi),
$$

where $t \mapsto h(t)$ is any horizontal lift in $H$ of the path $t \mapsto x(t)$ for the $G_{\gamma}$-connection $\bar{C}$, and where $\xi=\left(h\left(t_{0}\right)\right)^{-1}\left(z_{0}\right)$ is the unique element in $F$ such that $h\left(t_{0}\right)(\xi)=z_{0}$.

Remark 4. A $G_{\gamma}$-connection on a principal bundle $H\left(B, G, G_{\gamma}, \bar{H}\right)$ is a vector subbundle $\bar{C}$ of the tangent bundle $T H$, complementary to $\operatorname{ker} T \pi_{H}$, i.e. such that, for each $h \in H$,

$$
T_{h} H=\operatorname{ker} T_{h} \pi_{H} \oplus \bar{C}_{h},
$$

which remains invariant by the right action of $G_{\gamma}$ defined in Remark 3, i.e. which is such that for any $h \in H$ and $g \in G$,

$$
T R_{g}\left(\bar{C}_{h}\right)=\bar{C}_{h \circ g},
$$

where $T R_{g}: T H \rightarrow T H$ is the lift to vectors of the map $R_{g}: H \rightarrow H, h \mapsto h \circ g$. Conversely, a vector sub-bundle $\bar{C}$ of $T H$ complementary to $\operatorname{ker} T \pi_{H}$ which remains invariant by the right action of $G_{\gamma}$ is a $G$-connection on $H\left(B, G, G_{\gamma}, \bar{H}\right)$ : by using the $G$-invariance, one can prove that condition $(C)$ of Definition 1 is automatically satisfied.

The next proposition introduces the very important notion of connection form.

Proposition 2. Let $\bar{C}$ be a $G_{\gamma}$-connection on the principal bundle $H\left(B, G, G_{\gamma}, \bar{H}\right)$. There exists a unique 1-form $\omega$ on $H$, with values in the Lie algebra $\mathcal{G}$ of $G$, such that for all $h \in H$,

$$
\bar{C}_{h}=\left\{V \in T_{h} H ; \omega(V)=0\right\},
$$

and that for each $\eta \in \mathcal{G}$,

$$
\omega(\widehat{\eta}(h))=\eta .
$$


We have denoted by $\widehat{\eta}$ the fundamental vector field on $H$ associated to $\eta$, i.e. the vector field whose value, at an element $h \in H$, is

$$
\widehat{\eta}(h)=\left.\frac{d\left(R_{\exp (t \eta)} h\right)}{d t}\right|_{t=0} .
$$

The 1 -form $\omega$ is called the connection form of the $G_{\gamma}$-connection $\bar{C}$.

A connection on a principal bundle compatible with its structure group can be defined by its connection form, as shown by the next proposition.

Proposition 3. The connection form $\omega$ of a $G_{\gamma}$-connection $\bar{C}$ on the principal bundle $H\left(B, G, G_{\gamma}, \bar{H}\right)$ has the following properties:

(i) for each $\eta \in \mathcal{G}$ (the Lie algebra of $G$ ) and each $h \in H$,

$$
\omega(\widehat{\eta}(h))=\eta,
$$

where $\hat{\eta}$ is the fundamental vector field on $H$ associated to $\eta$;

(ii) for each $g \in G$, the pull-back $R_{g}^{*} \omega$ of the form $\omega$ by the right translation $R_{g}$ : $H \rightarrow H$, is

$$
R_{g}^{*} \omega=\operatorname{Ad}_{g^{-1}} \circ \omega .
$$

Conversely, any 1-form on $H$ which takes its values in the Lie algebra $\mathcal{G}$ of $G$, and which satisfies these two properties, is the connection form of a $G_{\gamma}$-connection on the principal bundle $H\left(B, G, G_{\gamma}, \bar{H}\right)$.

4. Cartan connections seen by Ehresmann. We will see in this section that connections on fibre bundles with a Lie group as structure group offer a very nice setting for a rigorous presentation of Cartan connections.

Proposition 4. Let $E(B, F, G, H)$ be a smooth bundle with a Lie group $G$ as structure group. We assume that $F$ is an homogeneous space $G / G^{\prime}$, with $G^{\prime}$ a closed subgroup of $G$. We denote by $o \in F$ the image of the unit element $e \in G$ by the projection $\pi_{G}: G \rightarrow F=G / G^{\prime}$. Moreover, we assume that there exists a smooth section $s_{0}: B \rightarrow E$ of the bundle $E(B, F, G, H)$. Let $H^{\prime}$ be the subset of elements $h \in H$ such that

$$
h(o)=s_{0}\left(\pi_{H}(h)\right) .
$$

Then $H^{\prime}$ is the total space of a principal bundle $H^{\prime}\left(B, G^{\prime}, G_{\gamma}^{\prime}, \overline{H^{\prime}}\right)$ with $G^{\prime}$ as structure group.

DEFINITION 4. Under the assumptions and with the notations of the previous proposition, let $\omega$ be the connection form of a $G$-connection on $H$, and let $\omega_{H^{\prime}}=i_{H^{\prime}}^{*} \omega$ be the 1-form induced on $H^{\prime}$ by $\omega$. We assume that for any $h^{\prime} \in H^{\prime}$ and any vector $V^{\prime} \in T_{h^{\prime}} H^{\prime}$, we have

$$
\omega_{H^{\prime}}\left(V^{\prime}\right)=\omega\left(V^{\prime}\right)=0 \text { if and only if } V^{\prime}=0 .
$$

Moreover, we assume that

$$
\operatorname{dim} B=\operatorname{dim} F .
$$

Such a connection on the bundle $E\left(B, G / G^{\prime}, G, H\right)$ (or on the associated principal bundle $\left.H\left(B, G, G_{\gamma}, \bar{H}\right)\right)$ is called a Cartan connection. 
REMARK 5. The assumptions and notations are those of Proposition 4 and Definition 4. Although defined on the total space $H^{\prime}$ of the principal bundle $H^{\prime}\left(B, G^{\prime}, G_{\gamma}^{\prime}, \overline{H^{\prime}}\right)$, the form $\omega_{H^{\prime}}$ is not a connection form on that bundle in the sense of Charles Ehresmann, since it takes its values in the larger Lie algebra $\mathcal{G}$, not in the Lie algebra $\mathcal{G}^{\prime}$ of $G^{\prime}$. However, the connection 1 -form $\omega$, defined on $H$, is fully determined by the form $\omega_{H^{\prime}}$ induced on $H^{\prime}$ : its values on a vertical vector tangent to $H$ (maybe not tangent to $H^{\prime}$ ) at a point of $H^{\prime}$ is known, since such a vector is the value of a fundamental vector field associated to an element of the Lie algebra $\mathcal{G}$; and its values at points of $H$ outside $H^{\prime}$ can be deduced from its values on $H^{\prime}$ by using the formula

$$
R_{g}^{*} \omega=\operatorname{Ad}_{g^{-1}} \circ \omega .
$$

Moreover, let $H^{\prime}\left(B, G^{\prime}, G_{\gamma}^{\prime}, \overline{H^{\prime}}\right)$ be a principal bundle whose structure group $G^{\prime}$ is a closed subgroup of a Lie group $G$, and let $\omega_{H^{\prime}}$ be a 1 -form defined on $H^{\prime}$, taking its values in the Lie algebra $\mathcal{G}$, satisfying the two properties:

(i) for each $\eta^{\prime} \in \mathcal{G}^{\prime}$ (the Lie algebra of $G^{\prime}$ ) and each $h^{\prime} \in H^{\prime}$,

$$
\omega_{H^{\prime}}\left(\widehat{\eta^{\prime}}\left(h^{\prime}\right)\right)=\eta^{\prime},
$$

where $\widehat{\eta^{\prime}}$ is the fundamental vector field on $H^{\prime}$ associated to $\eta^{\prime}$;

(ii) for each $g^{\prime} \in G^{\prime}$, the pull-back $R_{g^{\prime}}^{*} \omega_{H^{\prime}}$ of the form $\omega_{H^{\prime}}$ by the right translation $R_{g^{\prime}}: H^{\prime} \rightarrow H^{\prime}$, is

$$
R_{g^{\prime}}^{*} \omega_{H^{\prime}}=\operatorname{Ad}_{g^{\prime-1}} \circ \omega_{H^{\prime}}
$$

Given such a 1-form, called a Cartan connection form, we can build a principal bundle $H\left(B, G, G_{\gamma}, \bar{H}\right)$ with $G$ as structure group, and a connection form $\omega$ on $H$, in such a way that $H^{\prime}$ is a subset of $H$ and that $\omega_{H^{\prime}}$ is the form infuced on $H^{\prime}$ by the connection form $\omega$. We set $H=H^{\prime} \times{ }_{G^{\prime}} G$, quotient of the product $H^{\prime} \times G$ by the equivalence relation for which two elements $\left(h_{1}^{\prime}, g_{1}\right)$ and $\left(h_{2}^{\prime}, g_{2}\right)$ in $H^{\prime} \times G$ are equivalent if there exists $g^{\prime} \in G^{\prime}$ such that $h_{2}^{\prime}=R_{g^{\prime}}\left(h_{1}^{\prime}\right)$ and $g_{2}=\left(g^{\prime}\right)^{-1} g$. We identify $H^{\prime}$ with the set of equivalence classes of elements in $H^{\prime} \times\{e\}$, and we define $\omega$ on $H$ as indicated above.

REMARK 6. The first condition in the above definition,

$$
\omega\left(V^{\prime}\right)=0 \text { if and only if } V^{\prime}=0,
$$

implies $\operatorname{dim} B \leq \operatorname{dim} F$, since $\operatorname{dim} H^{\prime}=\operatorname{dim} B+\operatorname{dim} G^{\prime}$ and $\operatorname{dim} \mathcal{G}=\operatorname{dim} G=\operatorname{dim} F+$ $\operatorname{dim} G^{\prime}$. For a Cartan connection, the second condition in the above definition imposes the equality, $\operatorname{dim} B=\operatorname{dim} F$. When the first condition of the above definition is kept and the second condition is dropped, Ehresmann calls $\omega$ a "connexion de Cartan au sens large", i.e. a generalized Cartan connection.

REMARK 7. The existence of a Cartan connection implies that $H^{\prime}$ is parallelizable, since $\omega_{H^{\prime}}$ determines an isomorphism of $T H^{\prime}$ onto $H^{\prime} \times \mathcal{G}$.

The next proposition indicates another important consequence of the existence of a Cartan connection: the tangent bundle $T B$ to the base manifold is isomorphic to the bundle of vertical vectors, tangent to the fibres of the bundle $E(B, F, G, H)$ along the image of the global section $s_{0}$. 
Proposition 5. The assumptions and notations are those of Proposition 4 and Definition 4. Let $x \in B, w \in T_{x} B$. There exists a unique vector $\sigma(w) \in T_{s_{0}(x)} E_{x}$ such that, for any $h^{\prime} \in H_{x}^{\prime}$ and $W^{\prime} \in T_{h^{\prime}} H^{\prime}$ which satisfies $T \pi_{H^{\prime}}\left(W^{\prime}\right)=w$, we have

$$
\sigma(w)=T_{o} h^{\prime} \circ T_{e} \pi_{G} \circ \omega\left(W^{\prime}\right) .
$$

The map $\sigma: T B \rightarrow V_{s_{0}(B)} E$ so obtained is a vector bundle isomorphism, called the soldering isomorphism, of the tangent bundle $T B$ onto the vector bundle $V_{s_{0}(B)} E$ of vertical vectors, tangent to the fibres of $E$ along the image $s_{0}(B)$ of the global section $s_{0}$.

Proof. In the formula which defines $\sigma$ we have identified the Lie algebra $\mathcal{G}$ with the space $T_{e} G$, tangent to the Lie group $G$ at its unit element $e$. We have only to show that $T_{o} h^{\prime} \circ T_{e} \pi_{G} \circ \omega\left(W^{\prime}\right)$ depends only on $w=T \pi_{H^{\prime}}\left(W^{\prime}\right)$, not on the choices of $W^{\prime}$ and $h^{\prime}$. If we keep $h^{\prime}$ unchanged and replace $W^{\prime}$ by $W_{1}^{\prime}, W_{1}^{\prime}-W^{\prime}$ is the value at $h^{\prime}$ of a fundamental vector field associated to an element of the Lie algebra $\mathcal{G}^{\prime}$, therefore $\omega\left(W_{1}^{\prime}\right)-\omega\left(W^{\prime}\right) \in \mathcal{G}^{\prime}$ and $T_{e} \pi_{G} \circ \omega\left(W_{1}^{\prime}\right)=T_{e} \pi_{G} \circ \omega\left(W^{\prime}\right)$. Now if we replace $h^{\prime}$ by $h_{1}^{\prime}=h^{\prime} \circ g^{\prime}$, with $g^{\prime} \in G^{\prime}$, we may replace $W^{\prime}$ by $W_{1}^{\prime}=T R_{g^{\prime}}\left(W^{\prime}\right)$, so

$$
\begin{aligned}
T_{o} h_{1}^{\prime} \circ T_{e} \pi_{G} \circ \omega\left(W_{1}^{\prime}\right) & =T_{o}\left(h^{\prime} \circ g^{\prime}\right) \circ T_{e} \pi_{G} \circ \omega\left(T R_{g^{\prime}}\left(W^{\prime}\right)\right) \\
& =T_{o} h^{\prime} \circ T L_{g^{\prime}} \circ T_{e} \pi_{G} \circ \operatorname{Ad}_{g^{\prime}-1} \circ \omega\left(W^{\prime}\right) \\
& =T_{o} h^{\prime} \circ T_{e} \pi_{G} \circ \omega\left(W^{\prime}\right),
\end{aligned}
$$

since $T_{e} \pi_{G} \circ \operatorname{Ad}_{g^{\prime-1}} \circ \omega\left(W^{\prime}\right)=T L_{g^{\prime-1}} \circ \omega\left(W^{\prime}\right)$.

REMARK 8. For each point $x \in B$, the soldering isomorphism determined by a Cartan connection on the bundle $E\left(B, G / G^{\prime}, G, H\right)$ allows us to identify the space $T_{x} B$, tangent to the base $B$ at $x$, with the space $T_{s_{0}(x)} E_{x}$, tangent to the fibre $E_{x}$ at $s_{0}(x)$. It is in that precise sense that the soldering isomorphism allows us to consider that for each $x \in B$, the base manifold $B$ is tangent to the fibre $E_{x}$, the point $x \in B$ being in contact with the point $s_{0}(x) \in E_{x}$.

Definition 5. The assumptions are those of Definition 4. Let $t \mapsto x(t)$ be a smooth path in the base manifold $B$. The development of that path in the fibre $E_{x\left(t_{0}\right)}$ is the development (in the sense of Definition 2) of the smooth path in $E$,

$$
t \mapsto s_{0}(x(t)) .
$$

REmark 9. The development $t \mapsto y(t)$ of the path $t \mapsto x(t)$ in the fibre $E_{x\left(t_{0}\right)}$ can be obtained as follows. Let $t \mapsto h^{\prime}(t)$ be a smooth path in $H^{\prime}$ such that, for each $t$,

$$
\pi_{H^{\prime}}\left(h^{\prime}(t)\right)=x(t) .
$$

Such a path always exists (and is not unique). It is not horizontal for the connection $\bar{C}$ (except when $t \mapsto x(t)$ is constant) since $\omega_{H}$ does not vanish when applied to nonzero vectors tangent to $H^{\prime}$. Let $t \mapsto h(t)$ be the horizontal lift in $H$ of the path $t \mapsto x(t)$, through the point $h\left(t_{0}\right)=h^{\prime}\left(t_{0}\right)$. There exists a unique path $t \mapsto g(t)=h(t)^{-1} \circ h^{\prime}(t)$ in $G$ such that $g\left(t_{0}\right)=e$ and that, for each $t$,

$$
h^{\prime}(t)=h(t) \circ g(t) .
$$

An easy calculation shows that he development of $t \mapsto x(t)$ in $E_{x\left(t_{0}\right)}$ is the path

$$
t \mapsto y(t)=h^{\prime}\left(t_{0}\right) \circ g(t)(o),
$$


where $o \in G / G^{\prime}$ is the image of the unit element $e$ by the projection $\pi_{G}: G \rightarrow G / G^{\prime}$.

Moreover, the map

$$
\left.\left.\frac{d x(t)}{d t}\right|_{t=t_{0}} \mapsto \frac{d y(t)}{d t}\right|_{t=t_{0}}
$$

is the vector bundle isomorphism $\sigma$ of Proposition 5. That property is in agreement with the fact than when the fibre $E_{x\left(t_{0}\right)}$ is considered as tangent to the base $B$ at $x\left(t_{0}\right)$, the tangent vectors to the path $t \mapsto x(t)$ at $x\left(t_{0}\right)$ and to its development at $s_{0}\left(x\left(t_{0}\right)\right)$ should be related by the isomorphism $\sigma$.

\section{Examples of Cartan connections}

5.1. Homogeneous spaces. Let $G^{\prime}$ be a closed subgroup of a Lie group $G$ such that the left action of $G$ on the homogeneous space $F=G / G^{\prime}$ is effective. We consider the trivial $G$-bundle $E(B, F, G, H)$ with $B=F=G / G^{\prime}, E=B \times F=\left(G / G^{\prime}\right) \times\left(G / G^{\prime}\right)$, the fibres being the subsets $\{x\} \times F$ (in other words, the projection $\pi_{E}: B \times F \rightarrow B$ is the first projection). The associated principal $G$-bundle is the trivial bundle $H(B, G, G, \bar{H})$ with $H=B \times G$, an element $h=(x, g) \in H$ being considered as the diffeomorphism of the standard fibre $F$ onto the fibre $E_{x}=\{x\} \times F$ :

$$
y \mapsto(x, g(y)) .
$$

The structure group $G$ acts on $H$ on the right by

$$
\left((x, g), g^{\prime}\right) \mapsto\left(x, g g^{\prime}\right) .
$$

For the global section $s_{0}: B \rightarrow B \times F$, we choose the diagonal map $x \mapsto(x, x)$. The principal $G^{\prime}$-bundle $H^{\prime}\left(B, G^{\prime}, G_{\gamma}^{\prime}, \overline{H^{\prime}}\right)$ has as its total space $H^{\prime}$ the subset of elements $h^{\prime}=(x, g) \in H=B \times G$ which map the point $o=\pi_{G}(e)$ of $F$ into the image of the global section $s_{0}$, i.e. which are such that $x=g(o)$. Therefore $H^{\prime}$ can be identified with $G$ by means of $(g(o), g) \mapsto g$. The fibre of $H^{\prime}$ over a point $g(o)=\pi_{G}(g)$ of $B$ is the coset $g G^{\prime}$.

On the trivial bundle $B \times F$, we consider the integrable connection $C$ for which the parallel transport along any smooth path in $B$ with end points $x_{0}$ and $x_{1}$ is the map

$$
\left\{x_{0}\right\} \times F \rightarrow\left\{x_{1}\right\} \times F, \quad\left(x_{0}, y\right) \mapsto\left(x_{1}, y\right) .
$$

Given a smooth path $t \mapsto x(t)$ in $B$, its development in the fibre $\left\{x\left(t_{0}\right)\right\} \times F$ is $t \mapsto$ $\left(x\left(t_{0}\right), x(t)\right)$. The connection considered here being integrable, the soldering isomorphism integrates in the following sense: for each point $x$ in the base manifold $B$, there is a natural diffeomorphism $y \mapsto(x, y)$ of the base $B$ onto the fibre $\{x\} \times F$, whose differential at $x$ is the soldering isomorphism.

When we identify $H^{\prime}$ with $G$ as indicated above, the 1-form $\omega_{H^{\prime}}$ induced on $H^{\prime}$ by the connection form is the left Maurer-Cartan form on $G$ which, for any $g \in G$ and $X \in T_{g} G$, yields $\omega_{H^{\prime}}(X)=T L_{g^{-1}}(X)$; of course $\omega_{H^{\prime}}(X)=0$ if and only if $X=0$, as required by Definition 4.

5.2. Affine connections. Let $M$ be a smooth $n$-dimensional manifold. Its tangent bundle $T M$ is generally considered as a vector bundle, with the linear group $\operatorname{Gl}(n, \mathbb{R})$ as 
structure group; but here we consider it as an affine bundle, with the group $\operatorname{Aff}(n, \mathbb{R})$ of affine transformations of $\mathbb{R}^{n}$ as structure group. We set $G=\operatorname{Aff}(n, \mathbb{R}), G^{\prime}=\operatorname{Gl}(n, \mathbb{R})$; the standard fibre $G / G^{\prime}$ is identified with $\mathbb{R}^{n}$, considered as an affine space. The corresponding principal bundle $H\left(M, G, G_{\gamma}, \bar{H}\right)$ has as total space the set $H$ of affine isomorphisms of $\mathbb{R}^{n}$ onto the tangent space $T_{x} M$ at some point $x \in M$. We take as global section $s_{0}$ of $T M$ the zero section (for each $x \in M, s_{0}(x)$ is the zero vector at $x$ ). Let $H^{\prime}$ be the subset of elements in $H$ which map the origin of $\mathbb{R}^{n}$ into the image of $s_{0}$. In other words, an element $h^{\prime} \in H^{\prime}$ is a linear isomorphism of $\mathbb{R}^{n}$ onto the tangent space $T_{x} M$ (considered now as a vector space) at some point $x \in M$, and $H^{\prime}$ is the total space of the $G^{\prime}$-principal bundle $H^{\prime}\left(M, G^{\prime}, G_{\gamma}^{\prime}, \overline{H^{\prime}}\right)$.

Let $\bar{C}$ be a connection on the principal bundle $H\left(M, G, G_{\gamma}, \bar{H}\right)$. Its connection form $\omega$ takes its value in the Lie algebra of the affine group, which is a semi-direct product $\mathcal{G}=\mathcal{G}^{\prime} \times \mathbb{R}^{n}$ of the Lie algebra $\mathcal{G}^{\prime}$ of the linear group and of the Lie algebra $\mathbb{R}^{n}$ of the group of translations in $\mathbb{R}^{n}$. We may therefore write

$$
\omega=\omega^{\prime}+\omega^{\prime \prime},
$$

where $\omega^{\prime}$ takes its values in $\mathcal{G}^{\prime}$ and $\omega^{\prime \prime}$ in $\mathbb{R}^{n}$. In the same way, the form $\omega_{H^{\prime}}$ induced by $\omega$ on $H^{\prime}$ can be written

$$
\omega_{H^{\prime}}=\omega_{H^{\prime}}^{\prime}+\omega_{H^{\prime}}^{\prime \prime}
$$

where $\omega_{H^{\prime}}^{\prime}$ takes its values in $\mathcal{G}^{\prime}$ and $\omega_{H^{\prime}}^{\prime \prime}$ in $\mathbb{R}^{n}$.

We see that $\omega_{H^{\prime}}^{\prime}$ is a connection form on the $G^{\prime}$-principal bundle $H^{\prime}\left(M, G^{\prime}, G_{\gamma}^{\prime}, \overline{H^{\prime}}\right)$; in other words it is the connection form of a linear connection.

The second term $\omega_{H^{\prime}}^{\prime \prime}$ can be identified with a linear endomorphism $\sigma: T M \rightarrow T M$ as follows. Let $x \in M, h^{\prime} \in H_{x}^{\prime}, w \in T_{x} M$ and $W \in T_{h^{\prime}} H^{\prime}$ such that $T \pi_{H^{\prime}}(W)=w$. We set

$$
\sigma(w)=T_{e} h^{\prime} \circ w^{\prime \prime}(W) .
$$

As in the proof of Proposition 4, we see that $\sigma(w)$ only depends on $w$, not on the choices of $h^{\prime}$ and $W$, and that $\sigma$ is a linear endomorphism of the tangent bundle $T M$. Moreover, $\omega_{H^{\prime}}^{\prime \prime}$ is fully determined by $\sigma$.

Conversely, let $\varpi$ be the connection form of a linear connection, i.e. a connection on the principal bundle $H^{\prime}\left(M, G^{\prime}, G_{\gamma}^{\prime}, \bar{H}^{\prime}\right)$ of linear frames, and $\sigma$ be a linear endomorphism of $T M$. There exists a unique connection on the principal bundle $H\left(M, G, G_{\gamma}, \bar{H}\right)$ of affine frames whose connection form $\omega=\omega^{\prime}+\omega^{\prime \prime}$ is such that $\omega_{H^{\prime}}^{\prime}=\varpi$ and that the the linear endomrphism of $T M$ determined by $\omega_{H^{\prime}}^{\prime \prime}$ is $\sigma$. So a connection on the principal bundle of affine frames can be identified with a pair made by a linear connection and a linear endomorphism of $T M$.

REMARK 10. The Lie algebra of the affine group is a semi-direct product $\mathcal{G}=\mathcal{G}^{\prime} \times \mathbb{R}^{n}$ of the Lie algebra $\mathcal{G}^{\prime}$ of the linear group and the Lie algebra $\mathbb{R}^{n}$ of the group of translations $\mathbb{R}^{n}$. Let $\pi_{G}: G \rightarrow G / G^{\prime}$ be the canonical projection. When $\mathcal{G}$ is identified with $T_{e} G, G / G^{\prime}$ and $T_{o}\left(G / G^{\prime}\right)$ with $\mathbb{R}^{n}$, the second projection $\mathcal{G} \rightarrow \mathbb{R}^{n}$ is simply the tangent map $T_{e} \pi_{G}$ at the unit element. 
The connection form $\omega$ on the frame of affine bundles is a Cartan connection in the sense of Definition 4 if and only if $\omega_{H^{\prime}}$ has zero kernel, i.e. for each $h^{\prime} \in H^{\prime}, \bar{C}_{h^{\prime}} \cap T_{h^{\prime}} H^{\prime}=$ $\{0\}$. Let $S_{h^{\prime}}^{\prime}$ and $S_{h^{\prime}}^{\prime \prime}$ be the subspaces of $T_{h^{\prime}} H$ spanned by the values at $h^{\prime}$ of fundamental vector fields associated, respectively, to elements in the Lie subalgebras $\mathcal{G}^{\prime}$ and $\mathbb{R}^{n}$ of $\mathcal{G}$. We have the direct sum decompositions

$$
\operatorname{ker} T_{h^{\prime}} \pi_{H}=S_{h^{\prime}}^{\prime} \oplus S_{h^{\prime}}^{\prime \prime}, \quad T_{h^{\prime}} H=S_{h^{\prime}}^{\prime} \oplus S_{h^{\prime}}^{\prime \prime} \oplus \bar{C}_{h^{\prime}} .
$$

Since $\operatorname{ker} T_{h^{\prime}} \pi_{H^{\prime}}=T_{h^{\prime}} H^{\prime} \cap \operatorname{ker} T_{h^{\prime}} \pi_{H}=S_{h^{\prime}}^{\prime}$ we see that $\bar{C}_{h^{\prime}} \cap T_{h^{\prime}} H^{\prime}=\{0\}$ if and only if $T_{h^{\prime}} H^{\prime} \cap\left(S_{h^{\prime}}^{\prime \prime} \oplus \bar{C}_{h^{\prime}}\right)$ is the graph of a linear isomorphism of $C_{h^{\prime}}$ onto $S_{h^{\prime}}^{\prime \prime}$. Using the above remark, we conclude that the connection form $\omega$ is a Cartan connection if and only if the endomorphism $\sigma: T M \rightarrow T M$ determined by $\omega$ is an isomorphism. When that condition is fulfilled, $\sigma$ is the soldering isomorphism. It is very natural to impose to $\sigma$ to be the identity isomorphism of $T M$, since that ensures that any smooth path $t \mapsto x(t)$ in $M$ has, at a point $x\left(t_{0}\right)$, the same tangent vector as its development in $T_{x\left(t_{0}\right)} M$. Affine connections considered by É. Cartan [1] satisfy that property. Contrary to Ehresmann [8] and Lichnerowicz [15] who call affine connection any connection on the principal bundle of affine frames, Kobayashi and Nomizu [10] reserve that name for a Cartan connection whose soldering isomorphism is the identity of $T M$, and speak of generalized affine connections for all other connections on the principal bundle of affine frames.

5.3. Projective connections. In this subsection the standard fibre $F=\mathrm{P}(n, \mathbb{R})$ is the standard $n$-dimensional real projective space, quotient of $\mathbb{R}^{n+1}-\{0\}$ by the equivalence relation of colinearity. The structure group will be the corresponding projective linear group $G=\operatorname{PGl}(n, \mathbb{R})$, quotient of $\operatorname{Gl}(n+1, \mathbb{R})$ by the group of homotheties. We denote by $x_{0}, x_{1}, \ldots, x_{n}$ the standard coordinates in $\mathbb{R}^{n+1}$. Let $D$ be the straight line in $\mathbb{R}^{n+1}$ defined by the equations $x_{1}=\cdots=x_{n}=0, G^{\prime}$ be the closed subgroup of $G$ made by equivalence classes of elements $g \in \operatorname{Gl}(n+1, \mathbb{R})$ which map $D$ onto itself, and $o \in F$ be the image of $D-\{0\}$ by the projection $\mathbb{R}^{n+1}-\{0\} \rightarrow \mathrm{P}(n, \mathbb{R})$. The standard fibre $F=\mathrm{P}(n, \mathbb{R})$ will be identified with the homogeneous space $G / G^{\prime}$. For each $g \in \operatorname{Gl}(n, \mathbb{R})$, let $\widehat{g} \in \operatorname{PGl}(n, \mathbb{R})$ be the map

$$
\widehat{g}: \mathrm{P}(n, \mathbb{R}) \rightarrow \mathrm{P}(n, \mathbb{R}), \quad\left[x_{0}, x_{1}, \ldots, x_{n}\right] \mapsto\left[x_{0}, g\left(x_{1}, \ldots, x_{n}\right)\right] .
$$

The injective map $g \mapsto \widehat{g}$ will allow us to identify $\operatorname{Gl}(n, \mathbb{R})$ with a closed subgroup $G^{\prime \prime}$ of $\operatorname{PGl}(n, \mathbb{R})$, contained in the subgroup $G^{\prime}$ defined above.We observe that $G^{\prime}$ is the subgroup made by elements in $G$ which leave fixed the point $o \in F$, and that $G^{\prime \prime}$ is the subgroup made by elements which, in addition, leave globally invariant the hyperplane at infinity (image of the hyperplane defined by the equation $x_{0}=0$ by the projection $\left.\left(\mathbb{R}^{n+1}-\{0\}\right) \rightarrow \mathrm{P}(n, \mathbb{R})\right)$.

Now let $M$ be an $n$-dimensional smooth manifold. We consider the direct sum of the trivial bundle $M \times \mathbb{R}$ and of the tangent bundle $T M$. We take the complementary part of the image of the zero section in the total space of that bundle and quotient it by the equivalence relation of colinearity in the fibres. We obtain a fibre bundle $E(M, F)$ whose standard fibre is the projective space $F=\mathrm{P}(n, \mathbb{R})$. The tangent bundle can be 
considered as a dense subset of the total space $E$ of that bundle, if we identify, for each $x \in M$ and $v \in T_{x} M$, the vector $v$ with the element in $E_{x}$, equivalence class of $(1, v)$. Since the tangent bundle $T M$ and the trivial bundle $M \times \mathbb{R}$ admit as structure group, respectively, the linear group $G L(n, \mathbb{R})$ and the trivial group $\{e\}$, and since we have identified $\operatorname{Gl}(n, \mathbb{R})$ with a closed subgroup of $\operatorname{PGl}(n, \mathbb{R})$, the bundle $E(M, F)$ admits $G=\operatorname{PGl}(n, \mathbb{R})$ as structure group. Following Ehresmann's notations, we will denote it by $E(M, F, G, H)$, where $H$ is the principal bundle of projective frames in the fibres of $E(M, F)$.

For each $x \in M$, we denote by $s_{0}(x)$ the equivalence class of $\left(1,0_{x}\right)$, where $0_{x}$ is the zero vector at $x$. We may now define the subset $H^{\prime}$ of elements $h^{\prime} \in H$ which map the element $o \in F$ into the image of $s_{0}$, and observe that it is the total space of a principal bundle $H^{\prime}\left(M, G^{\prime}, G_{\gamma}^{\prime}, \overline{H^{\prime}}\right)$. A Cartan connection, in the sense of Definition 4, is a connection form $\omega$ on the principal bundle $H\left(M, G, G_{\gamma}, \bar{H}\right)$ such that the form $\omega_{H^{\prime}}$ induced on $H^{\prime}$ has zero kernel. The soldering isomorphism can still be considered as an isomorphism of the tangent bundle $T M$, since we have identified $T M$ with an open dense subset of $E$. A projective connection is a Cartan connection whose soldering isomorphism is the identity of $T M$. Cartan and Ehresmann have shown that projective connections exist on any manifold $M$.

5.4. Conformal connections. We follow the presentation of Kobayashi [11]. On the space $\mathbb{R}^{n+2}$, with coordinates $\left(x_{0}, x_{1}, \ldots, x_{n+1}\right)$, let $Q$ be the quadratic form

$$
Q\left(x_{0}, \ldots, x_{n+1}\right)=x_{1}^{2}+\cdots+x_{n+1}^{2}-x_{0}^{2} .
$$

Its signature is $(n+1,1)$. Let $\varnothing(n+1,1)$ be the subgroup of $\operatorname{Gl}(n+2, \mathbb{R})$ of elements which leave the quadratic form $Q$ unchanged, and let $Q_{0}$ be the cone, subset of $\mathbb{R}^{n+2}$, defined by the equation $Q=0$. Let us call rays the straight lines through the origin in $\mathbb{R}^{n+2}$. Any element $g \in \varnothing(n+1,1)$ maps a ray onto another ray, and a ray contained on $Q_{0}$ on another ray contained in $Q_{0}$. The set of rays is the projective space $\mathrm{P}(n+1, \mathbb{R})$. Therefore the group $O(n+1,1)$ acts on $\mathrm{P}(n+1, \mathbb{R})$ and that action leaves invariant the image $\mathcal{M}$ of $Q_{0}$ by the projection $\left(\mathbb{R}^{n+2}-\{0\}\right) \rightarrow \mathrm{P}(n+1, \mathbb{R})$. That action is transitive on $\mathcal{M}$. We denote by $o \in \mathcal{M}$ the ray defined by the equations $x_{0}-x_{n+1}=0, x_{1}=\cdots=x_{n}=0$, and by $G^{\prime} \subset \varnothing(n+1,1)$ its stabilizer. We will identify $\mathcal{M}$, which is called the Möbius space, with $\varnothing(n+1,1) / G^{\prime}$.

Let $\Pi$ be the affine hyperplane in $\mathbb{R}^{n+2}$ defined by the equation $x_{0}=1$. The affine injective map, defined on $\mathbb{R}^{n+1}$, with values in $\mathbb{R}^{n+2}$,

$$
\left(y_{1}, \ldots, y_{n+1}\right) \mapsto\left(x_{0}, x_{1}, \ldots, x_{n+1}\right),
$$

with

$$
x_{0}=1, x_{1}=y_{1}, \ldots, x_{n}=y_{n}, x_{n+1}=y_{n+1},
$$

has $\Pi$ as image and maps the sphere

$$
S^{n}=\left\{\left(y_{1}, \ldots, y_{n+1}\right) \in \mathbb{R}^{n+1} ; \sum_{i=1}^{n+1} y_{i}^{2}=1\right\}
$$

onto $Q_{0} \cap \Pi$. Since $\Pi$ meets each ray contained in $Q_{0}$ at a unique point, $Q_{0} \cap \Pi$ is 
diffeomorphic to $\mathcal{M}$. Therefore, by composition with the projection $\left(\mathbb{R}^{n+2}-\{0\}\right) \rightarrow$ $\mathrm{P}(n+1, \mathbb{R})$, we obtain a diffeomorphism of $S^{n}$ onto the Möbius space $\mathcal{M}$.

Let $\Pi_{1}$ be the affine hyperplane in $\mathbb{R}^{n+2}$ defined by the equation $x_{0}+x_{n+1}=1$. It meets each ray contained in $Q_{0}$ at a unique point, except the ray defined by the equations $x_{0}+x_{n+1}=0, x_{1}=\cdots=x_{n}=0$. The smooth injective map, defined on $\mathbb{R}^{n}$ and with values in $\mathbb{R}^{n+2}$,

$$
\left(z_{1}, \ldots, z_{n}\right) \mapsto\left(x_{0}, x_{1}, \ldots, x_{n+1}\right)
$$

with

$$
x_{0}=\frac{1+\sum_{i=1}^{n} z_{i}^{2}}{2}, x_{1}=z_{1}, \ldots, x_{n}=z_{n}, x_{n+1}=\frac{1-\sum_{i=1}^{n} z_{i}^{2}}{2},
$$

has $Q_{0} \cap \Pi_{1}$ as image. Composed with the projection $\left(\mathbb{R}^{n+2}-\{0\}\right) \rightarrow \mathrm{P}(n+1, \mathbb{R})$, that map yields a smooth injective map of $\mathbb{R}^{n}$ into the Möbius space $\mathcal{M}$, whose image is $\mathcal{M}$ minus one point, the missing point corresponding to the ray defined by the equations $x_{0}+x_{n+1}=0, x_{1}=\cdots=x_{n}=0$. The image, by that map, of the origin of $\mathbb{R}^{n}$ is the point $o \in \mathcal{M}$. Moreover, that map is equivariant with respect to the actions of the orthogonal group $\varnothing(n)$, on $\mathbb{R}^{n}$ and on $\mathbb{R}^{n+2}$, with the convention that an element $g \in \varnothing(n)$ is identified with the element $\widehat{g} \in \varnothing(n+1,1)$ which maps $\left(x_{0}, x_{1}, \ldots, x_{n}, x_{n+1}\right)$ on $\left(x_{0}, g\left(x_{1}, \ldots, x_{n}\right), x_{n+1}\right)$.

REMARK 11. When the Möbius space $\mathcal{M}$ is identified with the sphere $S^{n}$, the injective map $\mathbb{R}^{n} \rightarrow \mathcal{M}$ defined above is the inverse of the stereographic projection from $S^{n}$ minus its south pole onto $\mathbb{R}^{n}$.

Now let $(M, g)$ be an $n$-dimensional smooth Riemannian manifold. We consider the direct sum of two copies of the trivial bundle $M \times \mathbb{R}$ and of the tangent bundle $T M$, in the following order: $(M \times \mathbb{R}) \oplus T M \oplus(M \times \mathbb{R})$. To shorten the notation, we will denote that bundle by $\mathbb{R} \oplus T M \oplus \mathbb{R}$. Each element of the total space of that bundle is a triple $\left(v_{0}, v, v_{n+1}\right)$, with $v_{0}$ and $v_{n+1} \in \mathbb{R}$ and $v \in T M$, its projection on the base $M$ being the projection of $v$. Let $Q$ be the quadratic form, defined on the fibres of that bundle,

$$
Q\left(v_{0}, v, v_{n+1}\right)=g(v, v)+v_{n+1}^{2}-v_{0}^{2},
$$

and $Q_{0}$ be the subspace of $\mathbb{R} \oplus T M \oplus \mathbb{R}$ defined by the equation $Q=0$. We consider the projective bundle $\mathrm{P}(\mathbb{R} \oplus T M \oplus \mathbb{R})$, quotient of $(\mathbb{R} \oplus T M \oplus \mathbb{R}-\{0\})$ by the equivalence relation of colinearity in the fibres. We have denoted by $\{0\}$ the image of the zero section in the bundle $\mathbb{R} \oplus T M \oplus \mathbb{R}$. The image of $\left(Q_{0}-\{0\}\right)$ by the projection of $(\mathbb{R} \oplus T M \oplus \mathbb{R}-$ $\{0\})$ onto $\mathrm{P}(\mathbb{R} \oplus T M \oplus \mathbb{R})$ is the total space of a fibre bundle $E(M, \mathcal{M})$, with base the manifold $M$, and with standard fibre the Möbius space $\mathcal{M}$. The tangent bundle $T M$ can be considered as an open, dense subset of $E$ by identifying each vector $v \in T M$ with the equivalence class of $((1+g(v, v)) / 2, v,(1-g(v, v)) / 2)$. Since the manifold $M$ is equipped with a Riemannian metric, the tangent bundle $T M$ admits $\varnothing(n)$ as structure group. This is true for the bundle $E(M, \mathcal{M})$ too. But as seen above, $\varnothing(n)$ can be considered as a subgroup of $\varnothing(n+1,1)$, which acts on the standard fibre $\mathcal{M}$. The bundle $E(M, \mathcal{M})$ therefore has $G=\varnothing(n+1,1)$ as structure group and, using Ehresmann's notation, we 
will denote it by $E(M, \mathcal{M}, G, H)$. The total space $H$ of the corresponding principal bundle is the space of conformal frames on the Riemannian manifold $(M, g)$.

For each $x \in M$, we denote by $s_{0}(x)$ the equivalence class of $\left(1,0_{x}, 1\right)$, where $0_{x}$ is the zero vector at $x$. The subset $H^{\prime}$ of elements $h^{\prime} \in H$ which map the element $o \in \mathcal{M}$ into the image of $s_{0}$ is the total space of a principal bundle $H^{\prime}\left(M, G^{\prime}, G_{\gamma}^{\prime}, \overline{H^{\prime}}\right)$. A Cartan connection, in the sense of Definition 4 , is a connection form $\omega$ on the principal bundle $H\left(M, G, G_{\gamma}, \bar{H}\right)$ such that the form $\omega_{H^{\prime}}$ induced on $H^{\prime}$ has zero kernel. The soldering isomorphism can still be considered as an isomorphism of the tangent bundle $T M$, since we have identified $T M$ with an open dense subset of $E$. A conformal connection is a Cartan connection whose soldering isomorphism is the identity of $T M$. Cartan and Ehresmann have shown that conformal connections exist on any manifold $M$.

\section{Applications of connections.}

6.1. Gravitation. The first very important application of the notion of connection is probably to be found in the theory of General Relativity, in which the Levi-Civita connection associated to the pseudo-Riemannian structure on space-time plays a key role. According to the title of his paper [1], "Sur les espaces à connexion affine et la théorie de la relativité généralisée", É. Cartan was probably, for a large part, motivated by possible physical applications when he investigated the properties of connections. He explains how, in the framework of classical, non relativistic mechanics, a gravitation field (i.e. an acceleration field) can be included in the geometry of space-time by the use of an appropriate affine connection. We present here this idea in the more modern language of Ehresmann. Our manifold $M$ is the non relativistic space-time. For simplicity we assume that $\operatorname{dim} M=2$ (we take into account only one dimension for space). The choice of a Galilean frame and of units for time and length allows us to identify $M$ with $\mathbb{R}^{2}$, with coordinates $(t, x)$. The Lie group $G$, which will be called the affine Galileo group, is the group of affine transformations of $\mathbb{R}^{2}$ of the form

$$
g: \mathbb{R}^{2} \rightarrow \mathbb{R}^{2}, \quad(t, x) \mapsto\left(t^{\prime}=t+a, x^{\prime}=x+b+v t\right) .
$$

An element $g$ of $G$ is therefore a triple $(v, a, b)$, and the composition law of $G$ is

$$
\left(v_{2}, a_{2}, b_{2}\right)\left(v_{1}, a_{1}, b_{1}\right)=\left(v_{2}+v_{1}, a_{2}+a_{1}, b_{2}+b_{1}+v_{2} a_{1}\right) .
$$

Let $G^{\prime}$ be the linear Galileo group, i.e. the subgroup of elements of $G$ of the form $(v, 0,0)$. The homogeneous space $F=G / G^{\prime}$ can be identified with $\mathbb{R}^{2}$, with coordinates $(a, b)$. The bundle $E(M, F, G, H)$ is simply the tangent bundle $T M$, considered as an affine bundle, its structure group being restricted to the affine Galileo group (instead of the full affine group). The corresponding principal bundle $H\left(M, G, G_{\gamma}, \bar{H}\right)$ is the bundle of affine Galilean frames in $T M$. Its total space is simply the product $H=M \times G$. The subset of elements in $H$ which map the origin of $\mathbb{R}^{2}$ in the image of the zero section of $T M$ is $H^{\prime}=M \times G^{\prime}$. It is the total space of a principal bundle $H^{\prime}\left(M, G^{\prime}, G_{\gamma}^{\prime}, \overline{H^{\prime}}\right)$.

An affine Galilean connection is determined by a connection form $\omega$ on $H$, with values in the Lie algebra $\mathcal{G}$ of $G$, which induces on $H^{\prime}$ a form $\omega_{H^{\prime}}$ with zero kernel. In addition we impose to that form to be such that the corresponding soldering isomorphism is the 
identity of $T M$. The Lie algebra $\mathcal{G}$ can be identified with $\mathbb{R}^{3}$, by means of the basis $\left(\varepsilon_{v}, \varepsilon_{a}, \varepsilon_{b}\right)$ which corresponds to the coordinates $(v, a, b)$ on $G$. Taking into account the equivariance properties of $\omega$ and the fact that the corresponding soldering isomorphism is the identity of $T M$, we obtain

$\omega=(-V d t-W d x+d v) \varepsilon_{v}+(d t+d a) \varepsilon_{a}+(-(v+a V) d t+(1-a W) d x-v d a+d b) \varepsilon_{b}$, where $(t, x, v, a, b)$ are the coordinates on $H=M \times G, V$ and $W$ being two smooth functions on $M$ (therefore depending only on the coordinates $(t, x)$ ).

The development of the trajectory $t \mapsto(t, x(t))$ of a particle is a straight line if and only if the functions $V$ and $W$, on which the connection form depend, satisfy

$$
V(t, x(t))+W(t, x(t)) \frac{d x(t)}{d t}-\frac{d^{2} x(t)}{d t^{2}}=0 .
$$

We assume now that there exists on $M$ a gravity field $g$ (which may eventually depend on time $t$ and space location $x$ ). The equation of motion of a material particle submitted to that gravitational field is

$$
\frac{d^{2} x(t)}{d t^{2}}=g(t, x) .
$$

Therefore, if we choose $W=0$ and $V(t, x)=g(t, x)$, the development of the trajectory of any material particle submitted to the gravity field $g$, but to no other forces, is a straight line. When $g$ is a constant, the corresponding connection is integrable: a nonlinear change of coordinates in space-time eliminates the gravity force (in agreement with Einstein's thought experiment in which an observer in a lift in free fall no more feels the gravity force). This is no more true when $g$ is not constant. It would be interesting to develop the example in which $g$ is the acceleration field in Kepler's problem (in a non relativistic space-time of dimension 3); for a suitably chosen connection, the developments of the Keplerian trajectories of the planets should be straight lines.

6.2. Geometric quantization. Let $(M, \Omega)$ be a symplectic manifold. A prequantization of that symplectic manifold is a principal bundle $P\left(M, S^{1}, S^{1}, H\right)$ with base $M$ and with the circle $S^{1}$ as structure group, endowed with a connection 1-form $\omega$ whose curvature is $\Omega$.

Since the Lie algebra of $S^{1}$ can be identified with $\mathbb{R}$, the connection form $\omega$ can be considered as a contact form on $P$. According to a theorem by B. Kostant [12] and J.-M. Souriau [19], there exists a prequantization of $(M, \Omega)$ if and only if the cohomology class of $\Omega$ is integer.

6.3. Phases in Mechanics. Various uses of connections are made in the mathematical description of mechanical systems.

Let us consider a Hamiltonian system, depending of some parameters, which for any fixed value of these parameters, is completely integrable. The motion of the system, for a fixed value of the parameter, is quasi-periodic on a Lagrangian torus of phase space. At a certain time, the parameters vary slowly, describe a closed loop in the space of values of the parameters, and after taking again their initial values, remain constant. The motion of the system becomes again quasi-periodic on the same Lagrangian torus, but with a 
change of phase (the Hannay and Berry phase). This change of phase is interpreted as the holonomy of an Ehresmann connection in the works of Marsden, Montgomery and Ratiu [17, 18].

6.4. Nonholonomic constraints. Several different approaches have been used for the mathematical description of mechanical systems with constraints. In one of these approaches, the configuration space of the system is a smooth manifold and the constraints are described by a vector (or sometimes an affine) sub-bundle $C$ of the tangent bundle $T Q$. The admissible motions of the system are smooth curves $t \mapsto x(t)$ in $Q$ such that, for all $t$,

$$
\frac{d x(t)}{d t} \in C_{x(t)} .
$$

J. Koiller [13] considered systems where the configuration space $Q$ is the total space of a principal bundle over a base $B$, with a Lie group $G$ as structure group, and where the constraint $C$ is a connection on that principal bundle.

6.5. Active constraints. Let us consider a mechanical system in which some geometric constraints can be acted on, as a function of time, in order to control the motion of the system. For example, a cat in free fall can change the shape of her body to try (generally with success) to reach the ground on her feet.

For the mathematical description of such systems [16], we use a manifold $Q$ as configuration space, and a smooth submersion $\pi: Q \rightarrow S$ onto another manifold $S$ (the space of shapes of the cat's body, or more generally the space of possible states of the active constraint).

The dynamical properties of the system (other than those used to change the value of the active constraint) are described by a Lagrangian $L: T Q \rightarrow \mathbb{R}$. Let $V^{*} Q$ be the dual bundle of the vertical sub-bundle $V Q=\operatorname{ker} T \pi \subset T Q$. We can identify $V^{*} Q$ with the quotient bundle $T^{*} Q /(V Q)^{0}$. Let $\zeta: T^{*} Q \rightarrow V^{*} Q, q: V^{*} Q \rightarrow Q$ and $\widetilde{\pi}=\pi \circ q: V^{*} Q \rightarrow S$ be the projections. When the Lagrangian $L$ is

$$
L(v)=\frac{1}{2} g(v, v)-P(x), \quad \text { with } \quad x \in Q, v \in T_{x} Q,
$$

there is on the bundle $V^{*} Q \rightarrow S$ an Ehresmann connection (called the dynamical connection) which can be used to determine the way in which an infinitesimal change of the state of the active constraint, represented by a vector tangent to $S$, affects the motion of the mechanical system.

Let us call kinetic connection the Ehresmann connection, on the bundle $\pi: Q \rightarrow S$, for which the horizontal lift at $x \in Q$ of a vector $v \in T_{\pi(x)} S$ is the unique $w \in T_{x} Q$, orthogonal (with respect to $g$ ) to the vertical subspace $\operatorname{ker} T_{x} \pi$, such that $T \pi(w)=v$. The dynamic connection is characterized by the two properties:

(i) the horizontal lift at $z \in \widetilde{\pi}^{-1}(s)$ of a vector $v \in T_{s} S$ with respect to the dynamical connection projects on $Q$ onto the horizontal lift at $x=q(z)$ of $v$ with respect to the kinetic connection;

(ii) the horizontal lift to $V^{*} Q$ of any smooth vector field on $S$, with respect to the dynamical connection, is an infinitesimal automorphism of the Poisson structure of $V^{*} Q$. 
6.6. Maxwell's equations. The famous Maxwell's equations are usually written (see for example [9])

$$
\begin{aligned}
\operatorname{rot} \vec{E}+\frac{\partial \vec{B}}{\partial t} & =0, \quad \operatorname{rot} \vec{H}-\frac{\partial \vec{D}}{\partial t}=4 \pi \vec{j}, \\
\operatorname{div} \vec{B} & =0, \quad \operatorname{div} \vec{D}=4 \pi \rho,
\end{aligned}
$$

where $\vec{E}$ is the electric field, $\vec{B}$ the magnetic induction, $\vec{D}$ the diplacement current, $\vec{H}$ the magnetic field, $\rho$ the electric charge density and $\vec{j}$ the current density. Moreover there are constitutive equations which link $\vec{E}$ and $\vec{D}, \vec{B}$ and $\vec{H}$,

$$
\vec{D}=\varepsilon_{0} \vec{E}, \quad \vec{H}=\frac{1}{\mu_{0}} \vec{B} .
$$

Let us introduce the 2 -form on space-time (in which $\left(t, x^{1}, x^{2}, x^{3}\right)$ are the coordinates, with respect to some Galilean reference frame, some chosen units of time and length and some orthonormal frame in space)

$$
F=B_{1} d x^{2} \wedge d x^{3}+B_{2} d x^{3} \wedge d x^{1}+B_{3} d x^{1} \wedge d x^{2}+\left(E_{1} d x^{1}+E_{2} d x^{2}+E_{3} d x^{3}\right) \wedge d t,
$$

and

$$
G=D_{1} d x^{2} \wedge d x^{3}+D_{2} d x^{3} \wedge d x^{1}+D_{3} d x^{1} \wedge d x^{2}-\left(H_{1} d x^{1}+H_{2} d x^{2}+H_{3} d x^{3}\right) \wedge d t .
$$

Let us set

$$
J=\rho d x^{1} \wedge d x^{2} \wedge d x^{3}-\left(j_{1} d x^{2} \wedge d x^{3}+j_{2} d x^{3} \wedge d x^{1}+j_{3} d x^{1} \wedge d x^{2}\right) \wedge d t .
$$

Then we have

$$
G=\sqrt{\frac{\varepsilon_{0}}{\mu_{0}}} * F,
$$

where $*$ is the Hodge operator on the 4-dimensional pseudo-Riemannian manifold spacetime.

The velocity of light in vacuum is

$$
c=\sqrt{\frac{1}{\varepsilon_{0} \mu_{0}}} .
$$

Maxwell's equations become

$$
d F=0, \quad d G=4 \pi J, \quad \text { with } \quad G=\sqrt{\frac{\varepsilon_{0}}{\mu_{0}}} * F .
$$

Maxwell's equations can be made even more beautiful: the electromagnetic 2-form $F$ on space-time can be considered as the curvature form of a connection on a principal bundle, with space-time as base and the circle $S^{1}=\mathrm{U}(1)$ as structure group (this idea was first introduced by H. Weyl [20]). The 2-form $F$ should then be considered as taking its values in the Lie algebra $u(1)$ of $S_{1}$. The connection form $A$ such that $F=D A$, is not unique: we may add a closed 1-form (gauge transformation). The first Maxwell's equation, $d F=0$, is automatically satisfied. The second Maxwell's equation becomes

$$
D(* D A)=4 \pi \sqrt{\frac{\mu_{0}}{\varepsilon_{0}}} J,
$$

where $D$ is the covariant exterior differential operator. 
6.7. Yang-Mills fields. Gauge theories generalize Maxwell's theory of electromagnetism written in terms of connections. They use a principal bundle with space-time as base and a non-Abelian group as structure group $(\mathrm{U}(1) \times \mathrm{SU}(2) \times \mathrm{SU}(3)$ in the so-called standard model).

They introduce a connection 1-form $A$ on that bundle and lead, for the curvature form $F$ of the connection, to field equations similar to Maxwell's equations:

$$
D_{A} A=F, \quad D_{A} F=0 \quad \text { (Bianchi identity), }
$$

and

$$
D_{A} * F=J,
$$

where $D_{A}$ is the covariant differential with respect to the connection $A$ and $J$ a "current" which generalizes the 4-dimensional current density of Maxwell's theory.

7. Acknowledgements Many thanks to Paulette Libermann, for her very careful reading of this text and her many suggestions for improvements. And again my warmest thanks to the organizers and supporting institutions of this great International Conference.

\section{References}

[]1 É. CaRTAn, Sur les espaces à connexion affine et la théorie de la relativité généralisée, partie I, Ann. Ec. Norm., t. 40, 1923, p. 325-412.

[]2 É. Cartan, Sur les espaces à connexion affine et la théorie de la relativité généralisée, partie I (suite), Ann. Ec. Norm., t. 41, 1924, p. 1-25.

[]3 É. CaRTAn, Sur les espaces à connexion affine et la théorie de la relativité généralisée, partie II, Ann. Ec. Norm., t. 42, 1925, p. 17-88.

[]4 É. Cartan, Les espaces à connexion conforme, Ann. Soc. Pol. Math., t. 2, 1923, p. 171-221.

[15 É. CARTAn, Sur les variétés à connexion projective, Bull. Soc. Math. France, t. 52, 1924, p. 205-241.

[16 É. CaRTAn, Les récentes généralisations de la notion d'espace, Bull. Sc. Math., t. 48, 1924, p. 294-320.

[17 C. Ehresmann, Sur la théorie des espaces fibrés, Colloque de topologie algébrique du C.N.R.S., Paris, 1947, p. 3-15.

[]8 C. Ehresmann, Les connexions infinitésimales dans un espace fibré différentiable, Colloque de topologie de Bruxelles, 1950, p. 29-55.

[19 V. Guillemin and S. Sternberg, Symplectic techniques in physics, Cambridge University Press, Cambridge, 1984.

[]10 S. Kobayashi and K. Nomizu, Foundations of differential geeometry, vol. I, Interscience Publishers, New York, 1963.

[]11 S. Kobayashi, Transformation groups in differential geometry, Springer-Verlag, Berlin, 1972.

[]12 B. Kostant, Quantization and unitary representations, in Modern analysis and applications, Lecture Notes in Math. vol. 170, Springer-Verlag, New York, 1970, p. 101-184. 
[113 J. KoILleR, Reduction of some classical non-holonomic systems with symmetry, Arch. Ration. Mech. Anal., vol. 118, 1992, p. 113-148.

[]14 T. Levi-Civita, Rendiconti del Circ. matem. di Palermo, t. 42, 1917, p. 173-205.

[]15 A. Lichnerowicz, Théorie globale des connexions et des groupes d'holonomie, Edizioni Cremonese, Roma, 1962.

[]16 C.-M. MARLE, Géométrie des systèmes mécaniques à liaisons actives, in Symplectic Geometry and Mathematical Physics (P. Donato, J. Elhadad, G.M. Tuynman, editors), Birkhäuser, Boston, 1991, p. 260-287.

[17 J. Marsden, R. Montgomery and T. Ratiu, Reduction, Symmetry and Phases in Mechanics, Memoirs of the American Mathematical Society, 88 (1988), p. 269-294.

[18 R. Montgomery, The connection whose holonomy is the classical adiabatic angles of Hannay and Berry and its generalization to the non-integrable case, Commun. Math. Phys. 120 (1988), 269-294.

[19 J.-M. Souriau, Structure des systèmes dynamiques, Dunod, Paris, 1970.

[]20 H. WEYL, Temps, Espace, Matière (traduction française), Librairie scientifique Albert Blanchard, Paris, 1958. 